Sharif University of Technology
Scientia Iranica
SCIENTIA
I RAN ICA
http://scientiairanica.sharif.edu

\title{
Differential quadrature method for two-dimensional unsaturated water flow
}

\author{
A. Baghlani ${ }^{\mathrm{a}, *}$ and J. Nikzad ${ }^{\mathrm{b}}$ \\ a. Department of Civil and Environmental Engineering, Shiraz University of Technology, Shiraz, Iran. \\ b. Department of Engineering, Najaf Abad Branch, Islamic Azad University, Najaf Abad, Iran.
}

Received 13 September 2015; received in revised form 22 June 2016; accepted 25 September 2017

\author{
KEYWORDS \\ Unsaturated flow; \\ Differential \\ Quadrature Method \\ (DQM); \\ Richards' equation.
}

\begin{abstract}
This paper presents numerical solution of Richards' equation for water flow through unsaturated porous media. Differential Quadrature Method (DQM) is employed for the first time to solve the governing equations in two-dimensional space. The moisture content-based Richards' equation is considered. This equation is known as a highly nonlinear partial differential equation due to strong nonlinearity between hydraulic conductivity (and diffusivity) and moisture content. In order to investigate the robustness of DQM in dealing with such strong nonlinearities, two popular constitutive models, i.e. White and Broadbrige (1988) and Van Genuchten (1980), are investigated for the 2D case. The analytical solution based on Brooks and Coley model in a special 1D case is used to compare the results with those of DQM. For the 2D case, the study also demonstrates that DQM with considerably smaller number of grid points gives excellent results which are in close agreement with other numerical techniques such as multi-grid approach reported in the literature.
\end{abstract}

(C) 2018 Sharif University of Technology. All rights reserved.

\section{Introduction}

Prediction of fluid infiltration, such as water and pollutants, into unsaturated soil is of paramount importance in many fields. Transient flow in unsaturated zone is usually described using Richards' equation. Richards' equation is derived from combination of Darcy's equation and mass conservation law. Generally, Richards' equation may be written in three different forms, i.e. pressure-based in which pressure head, $h$, is considered as the dependent variable ( $h$-form), moisturecontent-based in which water content, $\theta$, is taken as the dependent variable ( $\theta$-form), and mixed form. Richards' equation is a nonlinear parabolic partial

*. Corresponding author. Fax: 0098 7137277656 E-mail addresses: baghlani@sutech.ac.ir (A. Baghlani); jamshidnikzad1364@gmail.com (J.Nikzad)

doi: $10.24200 /$ sci. 2017.4519 differential equation. Because of strong nonlinearity of the equation, analytical solutions are merely available for very special cases (e.g. [1-5]); hence, numerical approaches are preferable.

The most popular numerical approach to the simulation of saturated and unsaturated water flows in vadose zone is the finite difference method, (e.g. [6-10]). The finite-element method has been already employed to solve the governing equations for $1 \mathrm{D}$ and $2 \mathrm{D}$ flows through porous media (e.g., [11-14]). Recent studies show that finite volume method is also effective in discretization of Richards' equation (e.g. [15-19]). In addition to the aforementioned methods, other numerical approaches, such as multigrid method [20] and Backward Differentiation Formula (BDF), combined with exponential time integration approach, [21] have been developed for the simulation of unsaturated flow.

As an alternative numerical approach, Differential Quadrature Method (DQM) is a very effective tool to solve linear and nonlinear partial differential equations. 
As reported by Shu (2000) [22], DQM can also give very accurate numerical results using smaller number of grid points, hence requiring relatively low computational effort.

DQM has been already used to solve some problems in geo-science such as seepage analysis, dynamic behavior of fluid-saturated porous media, and consolidation (e.g. [23-25]). The review of related literature shows that in spite of its inherent capacity, DQM has not been utilized so far to simulate unsaturated water flow through porous media.

This paper aims to apply DQM for the first time to solve Richards' equation in two-dimensional space. Moisture-content-based equations are utilized for this purpose. In order to investigate the effectiveness of the method in dealing with highly nonlinear partial differential equations, two different constitutive models, including White and Broadbridge (1988) [26] and Van Genuchten (1980) [27], are studied. A smaller system of nonlinear equations, which arises after discretization of the equations, allows for the application of computationally effective iterative methods using DQM. In this study, instead of using computationally expensive Jacobian-based methods such as standard NewtonRaphson, a simple and effective iterative numerical method is proposed. A special case of the proposed 2D model in one-dimensional space is also presented in the results. 1D models are usually important since analytical solutions are available for comparison purposes. For the 2D case, two examples in five test cases are considered to investigate the effectiveness of the scheme. The results are in close agreement with those obtained by multigrid (MG) [20] approach previously published in the literature.

\section{Governing equations}

In this section, governing equations of the flow through unsaturated porous media in two-dimensional space are introduced. To complete the model, constitutive relations are also presented. The $1 \mathrm{D}$ model is a special case of the 2D model and is briefly presented in Section 4.

\subsection{Richards' equation}

Water flow in unsaturated soil is governed by Richards' equation. In this study, it is convenient to write the moisture-content form of this equation for homogeneous and isotropic soils as the normalized form, as in the following [20]:

$$
\frac{\partial u}{\partial t}-\nabla \cdot(D(u) \nabla u)+\frac{\partial K(u)}{\partial z}=0,
$$

where $x$ and $z$ are spatial coordinates such that $0 \leq$ $x \leq L_{x}, 0 \leq z \leq L_{z}$. The spatial coordinates can also be normalized by $L_{x}$ and $L_{z}$ to fall within the range
$[0,1]$. Vertical coordinate, $z$, is considered to be positive downward. Moreover, $D\left(L^{2} / T\right)$ is the hydraulic diffusivity, $K(L / T)$ is the hydraulic conductivity, and $u(x, z, t)$ is relative water content defined as follows:

$$
u=\frac{\theta-\theta_{r}}{\theta_{s}-\theta_{r}}
$$

where water content, $\theta\left(L^{3} / L^{3}\right)$, is defined within the range $\theta \in\left[\theta_{r}, \theta_{s}\right]$ in which $\theta_{r}\left(L^{3} / L^{3}\right)$ and $\theta_{s}\left(L^{3} / L^{3}\right)$ are the residual and saturated water contents, respectively. Eq. (1) in two-dimensional space can be written as follows:

$$
\frac{\partial u}{\partial t}-\frac{\partial}{\partial x}\left(D(u) \frac{\partial u}{\partial x}\right)-\frac{\partial}{\partial z}\left(D(u) \frac{\partial u}{\partial z}\right)+\frac{\partial K}{\partial z}=0
$$

\subsection{Constitutive relations}

For problem closure, some functional relationships between hydraulic conductivity and relative moisture content as well as between hydraulic diffusivity and relative water content should be established. In this study, two popular models, i.e. White and Broadbridge (1988) [26] and Van Genuchten (1980) [27], are investigated depending on the problem being solved.

\subsubsection{White and Broadbridge model}

White and Broadbridge (1988) [26] proposed the following relationships for hydraulic conductivity and diffusivity:

$$
\begin{array}{ll}
K(u)=\frac{(c-1) u^{2}}{(c-u)}, & c \in(1, \infty), \\
D(u)=\frac{c-(c-1)}{(c-u)^{2}}, & c \in(1, \infty),
\end{array}
$$

where $c$ is the model parameter.

\subsubsection{Van Genuchten model}

In Van Genuchten (1980) [27] model, the relationships concerning hydraulic conductivity and diffusivity are defined as follows:

$$
\begin{aligned}
K(u)= & \frac{K_{s}}{\theta_{s}-\theta_{r}} \sqrt{u}\left[1-\left(1-u^{\frac{1}{m}}\right)^{m}\right]^{2}, \\
D(u)= & \frac{(1-m) K_{s}}{\alpha m\left(\theta_{s}-\theta_{r}\right)} u^{\frac{1}{2}-\frac{1}{m}} \\
& {\left[\left(1-u^{\frac{1}{m}}\right)^{-m}+\left(1-u^{\frac{1}{m}}\right)^{m}-2\right], }
\end{aligned}
$$

where $K_{s}(L / T)$ is saturated conductivity, $m=1-1 / n$, and $\alpha\left(L^{-1}\right)$ and $n$ are shape parameters of the model. 


\section{Numerical solution approach}

The main objective of this study is to employ Differential Quadrature Method (DQM) for the first time to solve the governing equations described in the previous section. Differential quadrature method is a numerical approach to the solution of partial differential equations. It is known as an efficient method for both initial and boundary value problems, requiring remarkably small number of grid points. This method was first introduced by Bellman et al. (1972) [28]. In discretization method, the derivative of function, $f(x)$, with respect to $x$ for any grid point, $x_{i}$, is defined as a linear sum of function values in the entire field along a mesh line as:

$$
\begin{array}{r}
f_{x}\left(x_{i}\right)=\left.\frac{d f}{d x}\right|_{x=x_{i}}=\sum_{j=1}^{N} a_{i j} f\left(x_{j}\right), \\
\text { for } \quad i, j=1,2, \ldots, N,
\end{array}
$$

where $N$ is the number of grid points along a mesh line, and $a_{i j}$ represents the weighting coefficients. In DQM, the second-order derivative is written as follows:

$$
f_{x x}\left(x_{i}\right)=\left.\frac{d^{2} f}{d x^{2}}\right|_{x=x_{i}}=\sum_{j=1}^{N} b_{i j} f\left(x_{j}\right),
$$

$$
\text { for } \quad i, j=1,2, \ldots, N \text {, }
$$

where $b_{i j}$ represents the weighting coefficients for second-order derivative.

Two basic ideas in DQM are: i) computation of weighting coefficients; and ii) selection of spacing for grid points along a mesh line. There exist various methods to determine the weighting coefficients in DQM. In this study, Polynomial-based Differential Quadrature (PDQ) is adopted. Based on PDQ, weighting coefficients for the first-order derivative $\left(a_{i j}\right)$ and secondorder derivative $\left(b_{i j}\right)$ are obtained as follows [22]:

$$
\begin{array}{ll}
a_{i j}=\frac{M^{(1)}\left(x_{i}\right)}{\left(x_{i}-x_{j}\right) M^{(1)}\left(x_{j}\right)}, & \text { for } j \neq i, \\
a_{i i}=-\sum_{j=1}^{N} a_{i j}, & \text { for } j=i,
\end{array}
$$

where $M^{(1)}\left(x_{i}\right)$ and $M^{(1)}\left(x_{j}\right)$ are:

$$
\begin{aligned}
& M^{(1)}\left(x_{i}\right)=\prod_{j=1, j \neq i}^{N}\left(x_{i}-x_{j}\right), \\
& M^{(1)}\left(x_{j}\right)=\prod_{j=1, j \neq i}^{N}\left(x_{j}-x_{i}\right), \\
& b_{i j}=2 a_{i j}\left(a_{i i}-\frac{1}{x_{i}-x_{j}}\right), \quad \text { for } j \neq i,
\end{aligned}
$$

$$
b_{i i}=-\sum_{j=1, j \neq i}^{N} b_{i j}, \quad \text { for } j=i .
$$

It is worth pointing out that the location of grid points is crucial in DQM. Equally spaced grid points often yield very poor results and may destroy the numerical scheme. Herein, the position of each grid point in direction $x$ or $z$ is calculated using Chebyshev-GaussLobatto method:

$$
\begin{aligned}
& x_{i}=\frac{1}{2}\left[1-\cos \left(\frac{(i-1) \pi}{N_{x}-1}\right)\right] \times L_{x}, \\
& z_{j}=\frac{1}{2}\left[1-\cos \left(\frac{(j-1) \pi}{N_{z}-1}\right)\right] \times L_{z},
\end{aligned}
$$

where $N_{x}$ and $N_{z}$ represent the total number of grid points in $x$ and $z$ directions, respectively. Obviously, the total number of nodes $N_{t}$ in the grid is $N_{t}=N_{x} \times$ $N_{z}$.

For spatial and temporal discretizations of Eq. (3), by direct differentiating of Eq. (3), this equation is first rewritten as follows:

$$
\begin{aligned}
\frac{\partial u}{\partial t}= & \frac{\partial(D(u))}{\partial x} \cdot \frac{\partial u}{\partial x}+D(u) \frac{\partial^{2} u}{\partial x^{2}}+ \\
& \frac{\partial(D(u))}{\partial z} \cdot \frac{\partial u}{\partial z}+D(u) \frac{\partial^{2} u}{\partial z^{2}}-\frac{\partial K(u)}{\partial z} .
\end{aligned}
$$

A first-order finite difference scheme is used for temporal discretization of the LHS of Eq. (17):

$$
\left.\frac{\partial u}{\partial t}\right|_{z=z_{i}}=\frac{u_{i}^{n+1}-u_{i}^{n}}{\Delta t}
$$

where superscript $n$ denotes the time, and $\Delta t$ is the time step. follows:

The other terms are discretized using DQM as

$$
\begin{aligned}
& \frac{\partial K(u)}{\partial z}=\sum_{k=1}^{N_{z}} a_{j k} K(u)_{k}^{n+1}, \\
& \left.\frac{\partial u}{\partial x}\right|_{x=x_{i}}=\sum_{k=1}^{N_{x}} a_{i k} u_{k}^{n+1}, \\
& \left.\frac{\partial^{2} u}{\partial x^{2}}\right|_{x=x_{i}}=\sum_{k=1}^{N_{x}} b_{i k} u_{k}^{n+1}, \\
& \left.\frac{\partial u}{\partial z}\right|_{Z=Z_{i}}=\sum_{k=1}^{N_{z}} a_{j k} u_{k}^{n+1}, \\
& \left.\frac{\partial^{2} u}{\partial z^{2}}\right|_{Z=Z_{i}}=\sum_{k=1}^{N_{z}} b_{j k} u_{k}^{n+1},
\end{aligned}
$$




$$
\begin{aligned}
& \frac{\partial(D(u))}{\partial x}=\sum_{k=1}^{N_{x}} a_{i k} D(u)_{k}^{n+1}, \\
& \frac{\partial(D(u))}{\partial z}=\sum_{k=1}^{N_{z}} a_{j k} D(u)_{k}^{n+1} .
\end{aligned}
$$

Substituting Eqs. (18)-(25) into Eq. (17), the following discretized form is obtained:

$$
\begin{aligned}
\frac{u_{i}^{n+1}-u_{i}^{n}}{\Delta t}= & {\left[\left(\sum_{k=1}^{N_{x}} a_{i k} D(u)_{k}^{n+1}\right.\right.} \\
& \left.\times \sum_{k=1}^{N_{x}} a_{i k} u_{k}^{n+1}+D(u) \sum_{k=1}^{N_{x}} b_{i k} u_{k}^{n+1}\right) \\
& +\left(\sum_{k=1}^{N_{z}} a_{j k} D(u)_{k}^{n+1} \times \sum_{k=1}^{N_{z}} a_{j k} u_{k}^{n+1}\right. \\
& \left.+D(u) \sum_{k=1}^{N_{z}} b_{j k} u_{k}^{n+1}\right) \\
& \left.-\sum_{k=1}^{N_{z}} a_{j k} K(u)_{k}^{n+1}\right]
\end{aligned}
$$

Rearranging Eq. (26) gives:

$$
\begin{aligned}
u_{i}^{n+1}-\Delta t= & {\left[\left(\sum_{k=1}^{N_{x}} a_{i k}(D(u))_{k}^{n+1}\right.\right.} \\
& \left.\times \sum_{k=1}^{N_{x}} a_{i k} u_{k}^{n+1}+D(u) \sum_{k=1}^{N_{x}} b_{i k} u_{k}^{n+1}\right) \\
& +\left(\sum_{k=1}^{N_{z}} a_{j k}(D(u))_{k}^{n+1} \times \sum_{k=1}^{N_{z}} a_{j k} u_{k}^{n+1}\right. \\
& \left.\left.+D(u) \sum_{k=1}^{N_{z}} b_{j k} u_{k}^{n+1}\right)\right] \\
& -\Delta t \sum_{k=1}^{N_{z}} a_{i k} K(u)_{k}^{n+1}=u_{i}^{n} .
\end{aligned}
$$

In this equation, $i$ and $k$ represent the locations of nodes in $x$ and $z$ directions, respectively. In its matrix form, the system of Eq. (27) can be written as follows:

$$
\left[M(u)^{n+1}\right]_{N t \times N t}\left\{\mathbf{U}^{n+1}\right\}_{N t \times 1}=\left\{\mathbf{U}^{n}\right\}_{N t \times 1},
$$

where $M$ represents $N_{t} \times N_{t}$ coefficient matrix and $\boldsymbol{U}$ denotes the vector of unknown variables. Obviously, matrix $M$ entails DQM coefficients $a_{i j}$ and $b_{i j}$, hydraulic and diffusivity conductivity as well as time step
$\Delta t$. The RHS of Eq. (28) is known from the previous time step.

Any standard numerical method, such as NewtonRaphson, can be used to solve the system of nonlinear Eq. (28). Some iterative methods, such as modified Picard iterations, have been already employed to solve the nonlinear system of equations arising from discretization of Richards' equation [29]. In an attempt to avoid Picard/Newton iterations, Jancu et al. employed a nonlinear Multi Grid (MG) approach to solving 2D Richards' equation. Their method was based on second-order accurate time discretization combined with a second-order accurate finite difference spatial discretization. One of the main advantages of DQM, compared with other numerical methods, is that a coarser grid with fewer grid points can be used to achieve the same accuracy in the results. Numerical examples show that the order of matrix $M$ is small compared with the coefficient matrices resulting in discretization of equations with other iterative numerical methods such as nonlinear multigrid (MG) method. This permits a simpler iterative method to be used, eliminating time-consuming computation of the Jacobian matrix required in Newton's method. The current method, as Eq. (26) reveals, is based on a first-order accurate time discretization combined with DQM for spatial discretization. The resulting equations can be iteratively solved via the following approach. Eq. (28) is rewritten as follows:

$$
\left[M(u)^{n+1, m}\right]_{N t \times N t}\left\{\mathbf{U}^{n+1, m+1}\right\}_{N t \times 1}=\left\{\mathbf{U}^{n}\right\}_{N t \times 1},
$$

where $m$ denotes iteration number.

To solve the system of Eq. (29), first, the numbers of grid points in each direction are chosen and the locations of grid points are calculated using Eq. (16). By using the location of grid points, the values of $a_{i j}$ and $b_{i j}$ are calculated according to PDQ described in this section. Secondly, $m$ and $n$ are set to 0 , and an initial guess $\mathbf{U}_{0}$ is assigned to $\mathbf{U}$. Since the initial $\mathbf{U}_{0}$ guess affects the convergence of the scheme in the solution of nonlinear equations by iterative methods, the initial conditions of the problem are used to assign appropriate values to $\mathbf{U}_{0}$. Then, depending on the constitutive model used, the values of hydraulic conductivity and diffusivity for specified $\mathbf{U}_{0}$ are calculated (Eqs. (4)-(7)). Subsequently, the values of $\mathbf{U}^{n+1, m+1}$ for the next iteration can be obtained using Eq. (29):

$$
\left\{\mathbf{U}^{n+1, m+1}\right\}=\left[M(u)^{n+1, m}\right]^{-1}\left\{\mathbf{U}^{n}\right\}
$$

The iterations continue until the difference between the norms of the two successive solutions is less than a specified error, $\varepsilon$, that is:

$$
a b s\left(\left\|U^{m+1}\right\|-\left\|U^{m}\right\|\right) \leq \varepsilon .
$$


In the current study, a value of $\varepsilon=0.5 \times 10^{-6}$ is adopted. Once the iterations are completed, we march in time and computations are accomplished for the next time step in a similar fashion.

\section{Numerical examples}

In order to investigate the robustness of the aforementioned numerical scheme, both $1 \mathrm{D}$ and $2 \mathrm{D}$ problems are solved. In the $1 \mathrm{D}$ case, the numerical solutions can be compared with available analytical solutions. For the 2D case, two problems, including five test cases, are considered. Each problem employs a different constitutive model and consists of different soil types. Various types of boundary conditions, including Dirichlet, Neumann, and Robin boundary conditions, are also investigated. These test cases have been chosen since they have been already solved numerically using Multi Grid (MG) method by Juncu et al. (2010) [20].

\section{1. $1 D$ case}

For the nonlinear infiltration problem, analytical solutions are available for simplified problems in the 1D case; such analytical solutions are valuable for the verification of numerical solutions. Asghari et al. (2011) [30] proposed an analytical solution to 1D Richards' equation based on exp-function. Here, a brief review of the $1 \mathrm{D}$ model, which is a simplified version of our 2D model, is first presented, and then the results of employing the proposed DQM approach in the 1D case are compared with those of the aforementioned analytical solution.

The one-dimensional version of Eq. (3) is:

$$
\frac{\partial u}{\partial t}-\frac{\partial}{\partial z}\left(D(u) \frac{\partial u}{\partial z}\right)+\frac{\partial K(u)}{\partial z}=0
$$

To obtain the analytical solution in [30], the model of Brooks and Corey [31] was used for soil parameters as follows:

$$
\begin{array}{ll}
D(u)=D_{0}(n+1) u^{m}, & m \geq 0, \\
K(u)=K_{0} u^{k}, & k>1,
\end{array}
$$

where $D_{0}, K_{0}, k, n$, and $m$ are constants representing soil properties.

For $m=0, k=2, K_{0}=0.5$, and the following initial conditions:

$$
\theta(z, 0)=\frac{1}{2}\left(1+\tanh \left(-\frac{z}{4}\right)\right) \text {. }
$$

Asghari et al. [30] obtained the following analytical solution:

$$
\theta(z, t)=\frac{1}{2}\left(1+\tanh \left(-\frac{z}{4}+\frac{t}{8}\right)\right)
$$

Figure 1 compares analytical solutions and DQM numerical model at time $t=3$ and $t=6$. As a measure

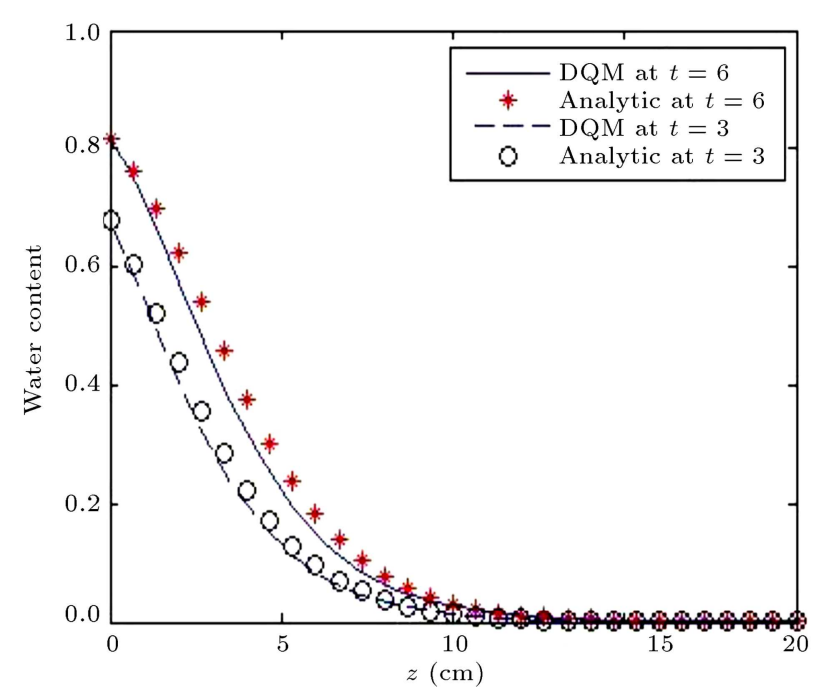

Figure 1. Comparison of DQM results and analytical solutions for $1 \mathrm{D}$ case at $t=3$ and $t=6$.

for the differences between model prediction and analytical solution, the standard Root-Mean-Square-Error (RMSE) is adopted. Consequently, RMSE $=0.0436$ at time $t=3 \mathrm{hr}$ and RMSE $=0.0671$ at time $t=6 \mathrm{hr}$ are obtained. As the results show, the proposed DQM yields very satisfactory results for the $1 \mathrm{D}$ case.

\section{2. $2 D$ case}

For the two-dimensional case, two different constitutive models are considered. The aforementioned numerical procedure has been used to find the saturation profiles in different times. In all test cases, after fulfillment of the stopping criteria, i.e. Eq. (31), the discrete norm of the residuals is approximately $2.27 \times 10^{-14}$, showing that discrete nonlinear equations have been acceptably satisfied in each iteration. The problems are as follows.

\subsubsection{Problem 1: White and Broadbridge model}

In the first problem of the $2 \mathrm{D}$ case, the White and Broadbridge constitutive model [26] in three soil types, ranging from highly nonlinear to weakly nonlinear soil, is investigated. For all cases, the problem is solved by DQM using $N_{x}=21$, and $N_{z}=40$ grid points in $x$ and $z$ directions, respectively. A finer grid did not cause any significant improvement in the results. Furthermore, time step $\Delta t=0.01 \mathrm{hr}$ is adopted.

The parameters' values as well as initial and boundary conditions used to simulate the three cases of this problem are as follows:

- Case 1. Highly nonlinear soil:

$$
\begin{aligned}
& L_{x}=1 \mathrm{~m}, \quad L_{z}=4 \mathrm{~m}, \quad c=1.01, \\
& t=0, \quad u=0, \quad z=0, \\
& K(u)-D(u) \cdot \frac{\partial u}{\partial z}=2 K(u)+\gamma_{0}(x),
\end{aligned}
$$




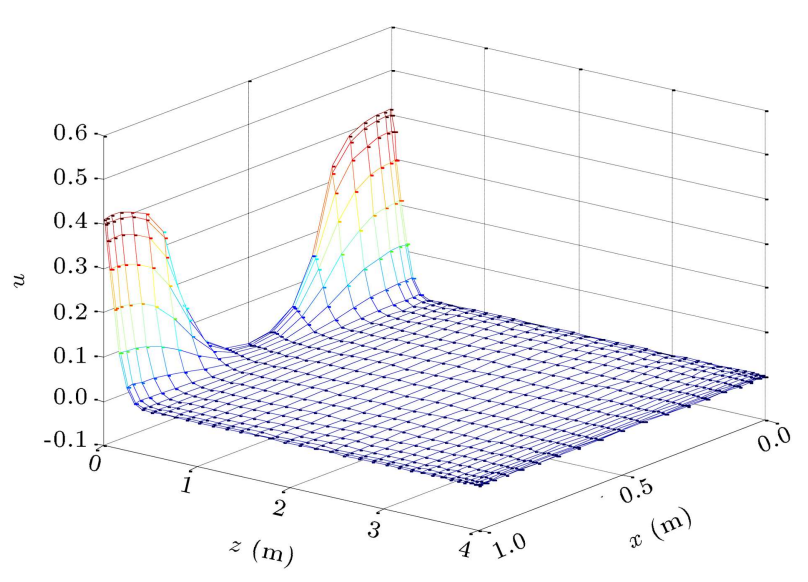

(a)

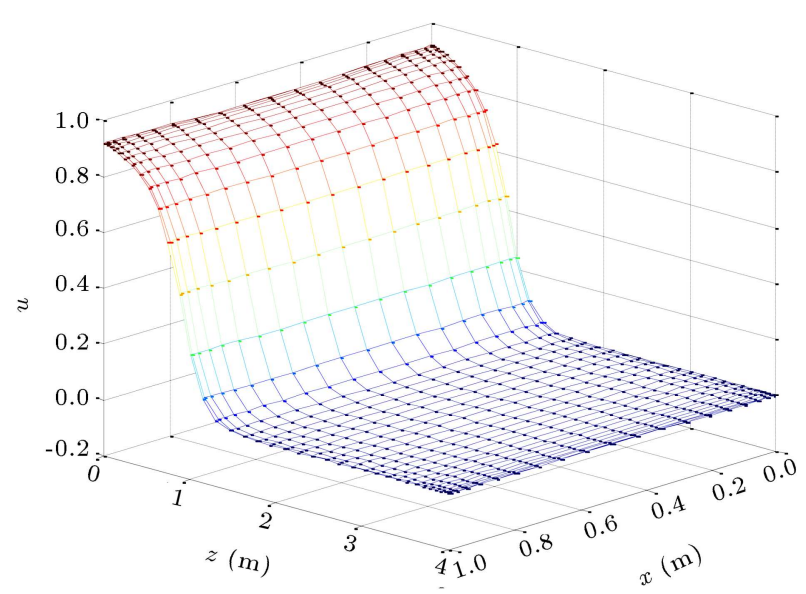

(b)

Figure 2. Saturation profiles for problem 1, Case 1 (highly nonlinear soil) using White and Broadbridge model by DQM at (a) $t=1 \mathrm{hr}$ and (b) $t=14.51 \mathrm{hr}$.

$$
\begin{aligned}
& \gamma_{0}(x)=\left\{\begin{array}{l}
0.05, \quad x<0.25 \text { or } x>0.75 \\
0.0, \quad 0.25 \leq x \leq 0.75
\end{array}\right. \\
& z=L_{z}, \quad u=0, \quad x=0, \text { and } \\
& x=L_{x}, \quad \frac{\partial u}{\partial x}=0 .
\end{aligned}
$$

Figure 2(a) and (b) show saturation profiles obtained by DQM for water infiltration in the highly nonlinear soil at times $t=1 \mathrm{hr}$ and $t=14.51 \mathrm{hr}$, respectively. The figures are in very close agreement with those obtained by using MG [20]. It is worthy to point out that $\mathrm{MG}$ converges to accurate results by employing a $64 \times 256$ grid. However, DQM needs only a $21 \times$ 40 grid to converge to the same results. Hence, a much coarser grid is required for DQM which is more effective with regard to computational cost.

- Case 2. Moderately nonlinear soil:

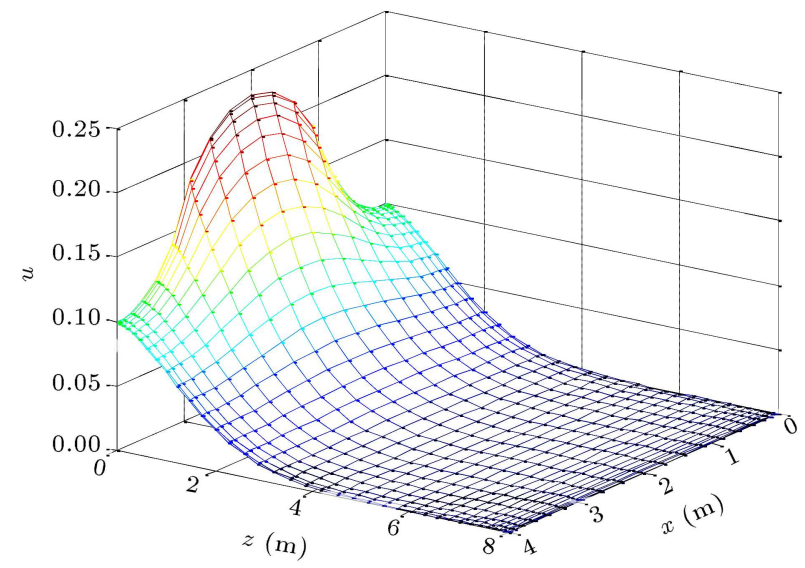

Figure 3. Saturation profiles for problem 1, Case 2 (moderately nonlinear soil) using White and Broadbridge model by DQM at $t=6 \mathrm{hr}$.

$$
\begin{aligned}
& L_{x}=4 \mathrm{~m}, \quad L_{z}=8 \mathrm{~m}, \quad c=1.48, \\
& t=0, \quad u=0, \quad z=0, \\
& K(u)-D(u) \cdot \frac{\partial u}{\partial z}=2 K(u)+\gamma_{0}(x), \\
& \gamma_{0}(x)=\left\{\begin{array}{c}
0.0, \quad x<1 \quad \text { or } \quad x>3 \\
0.05, \quad 1<x<3
\end{array}\right. \\
& z=L_{z}, \quad u=0, \quad x=0, \\
& x=L_{x}, \quad \frac{\partial u}{\partial x}=0 .
\end{aligned}
$$

Figure 3 shows saturation profiles for water infiltration in the moderately nonlinear soil obtained by DQM at time $t=6 \mathrm{hr}$. Both MG [20] and DQM lead to the same results. However, $\mathrm{MG}$ needs a $256 \times 512$ grid whereas a $21 \times 40$ grid is sufficient for DQM.

Case 3. Weakly nonlinear soil:

$$
\begin{aligned}
& L_{x}=1 \mathrm{~m}, \quad L_{z}=8 \mathrm{~m}, \quad c=1.48, \\
& t=0, \quad u=0, \quad z=0, \\
& K(u)-D(u) \cdot \frac{\partial u}{\partial z}=2 K(u)+\gamma_{0}(x), \\
& \gamma_{0}(x)= \begin{cases}0.00, & x \leq 0.25 \text { or } \quad x \geq 0.75 \\
0.05, & 0.25<x<0.75\end{cases} \\
& z=L_{z}, \quad u=0, \quad x=0, \quad \text { and } \\
& x=L_{x}, \quad \frac{\partial u}{\partial x}=0 .
\end{aligned}
$$

Figure 4 gives the results obtained by DQM at time $t=1 \mathrm{hr}$ for this case. Similar to previous cases, 


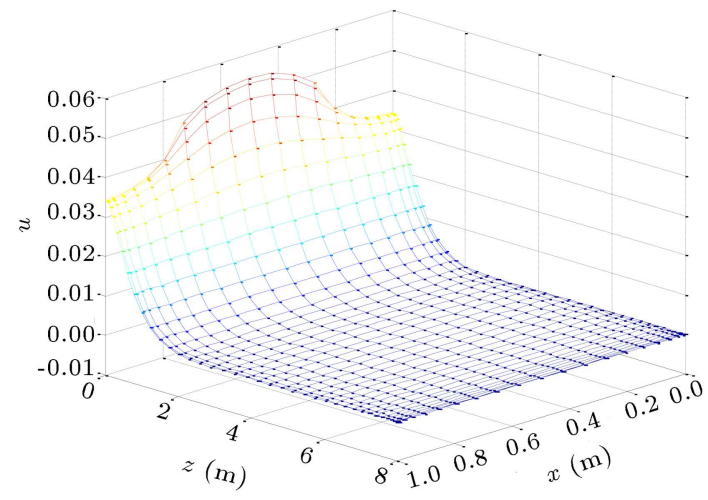

Figure 4. Saturation profiles for problem 1, Case 3 (weakly nonlinear soil) using White and Broadbridge model by DQM at $t=1 \mathrm{hr}$.

very satisfactory results are also obtained for this case with a coarser mesh using DQM, compared with MG [20].

\subsubsection{Problem 2: Van Genuchten model}

A more complicated constitutive model of Van Genuchten (1980) [27] for two different soils is studied in this problem. In both cases, the problem is solved by DQM, using $N_{x}=26$, and $N_{z}=26$ grid points in each direction. Moreover, time step $\Delta t=0.01 \mathrm{hr}$ is adopted.

The parameters values, initial and boundary conditions used for this problem are as follows:

- Case 1. New Mexico soil:

$$
\begin{aligned}
& L_{x}=1 \mathrm{~m}, \quad L_{z}=1 \mathrm{~m}, \\
& t=0, \quad u=0.1, \quad z=0, \\
& K(u)-D(u) \cdot \frac{\partial u}{\partial z}=2 K(u)+\gamma_{0}(x) \\
& \gamma_{0}(x)=\left\{\begin{array}{l}
0.00, x \leq 0.25 \text { or } \quad x \geq 0.75 \\
0.003,0.25<x<0.75
\end{array}\right. \\
& z=L_{z}, \quad u=0.1, \quad x=0, \quad \text { and } \\
& x=L_{x}, \quad \frac{\partial u}{\partial x}=0 .
\end{aligned}
$$

Other parameters for simulation are reported in Table 1.

Table 1. Parameters used in problem 2 for two cases.

\begin{tabular}{ccccccc}
\hline No. & $\boldsymbol{\theta}_{\boldsymbol{r}}$ & $\boldsymbol{\theta}_{\boldsymbol{s}}$ & $\boldsymbol{\alpha}$ & $\boldsymbol{n}$ & $\boldsymbol{m}$ & $\boldsymbol{K}_{\boldsymbol{s}}$ \\
\hline Case 1 & 0.102 & 0.368 & 3.35 & 2 & 0.5 & 0.332083 \\
Case 2 & 0.058 & 0.473 & 0.58 & 1.6745 & 0.40280 & 0.012825 \\
\hline
\end{tabular}

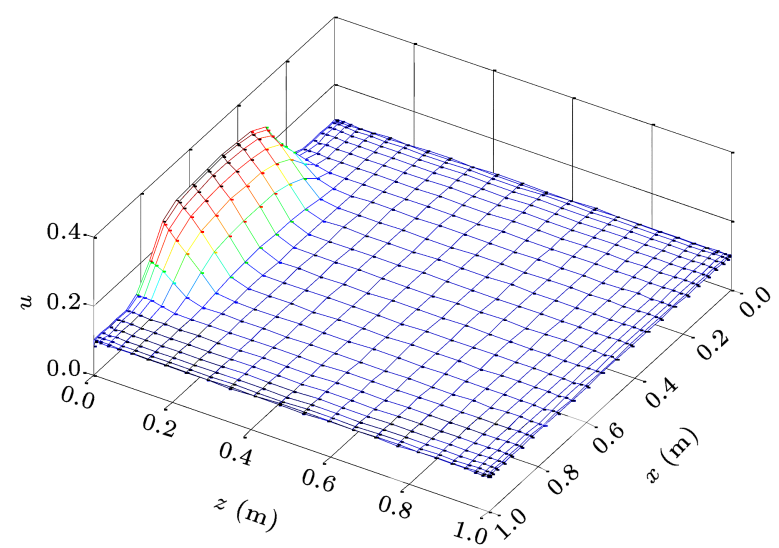

(a)

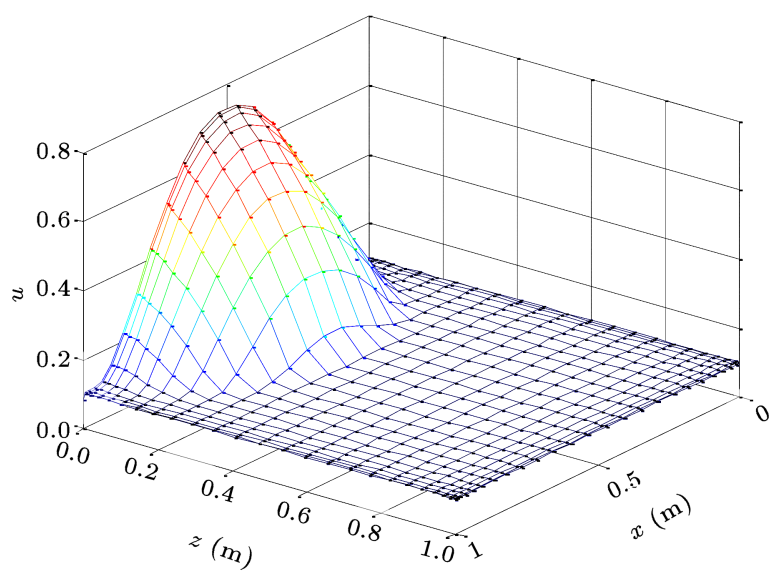

(b)

Figure 5. Saturation profiles for problem 2, Case 1 (New Mexico soil) using Van Genuchten model by DQM at (a) $t=4 \mathrm{hr}$ and (b) $t=9.83 \mathrm{hr}$.

Figure 5(a) and (b) give the saturation profiles obtained by DQM at times $t=4 \mathrm{hr}$ and $t=$ $9.83 \mathrm{hr}$ respectively, for this case. In spite of using a complicated highly nonlinear constitutive model for this test case, very satisfactory results are achieved, suggesting the robustness of the scheme.

- Case 2. Travelling wave in silt:

$$
\begin{aligned}
& L_{x}=1 \mathrm{~m}, \quad L_{z}=1 \mathrm{~m}, \quad t=0, \quad u=0.1, \\
& z=0, \quad u=0.8, \quad z=L_{z}, \quad u=0.1, \\
& x=0, \quad \text { and } \quad x=L_{x}, \quad \frac{\partial u}{\partial x}=0 .
\end{aligned}
$$

Other parameters for simulation are reported in Table 1.

In Figure 6(a), (b), and (c), the saturation profiles for three different times of $t=1 \mathrm{hr}, t=10$ $\mathrm{hr}$, and $t=30 \mathrm{hr}$, obtained by DQM are presented, respectively. The agreement between the results found by MG [20] is very satisfactory in this problem as well. 


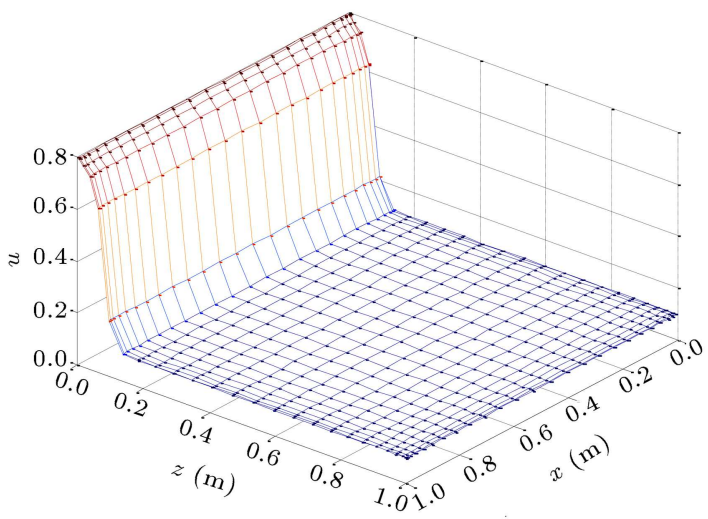

(a)

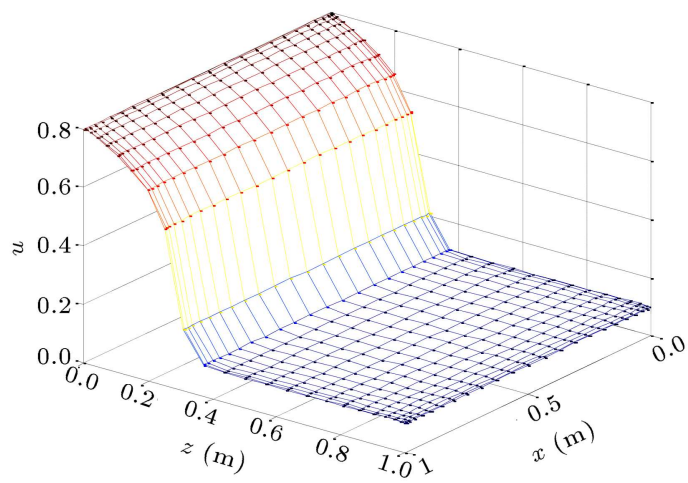

(b)

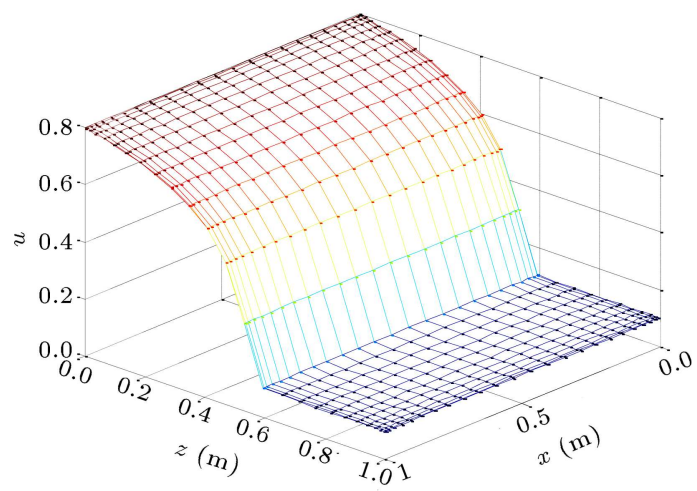

(c)

Figure 6. Saturation profiles for problem 2, Case 2 (traveling wave in silt) using Van Genuchten model by DQM at (a) $t=1 \mathrm{hr}$, (b) $t=10 \mathrm{hr}$, and (c) $t=30 \mathrm{hr}$.

\section{Conclusion}

In this study, numerical solution of water flow through unsaturated porous media in two-dimensional space was presented. For this purpose, the saturated form of Richards' equation was solved by Differential Quadrature Method (DQM). Two different constitutive models were incorporated into the governing equations in the $2 \mathrm{D}$ case. The simplified $1 \mathrm{D}$ case was also presented, and the results were compared with those of the available analytical solutions. For the two-dimensional case, two problems in five test cases were investigated. The study shows that DQM requires fewer grid points, compared with MG, which consequently leads to a very smaller system of nonlinear equations. Therefore, in terms of computational effort, more effective iterative methods, rather than traditional Jacobian-based methods, can be employed to solve the resulting system of equations. The results of using DQM with much coarser grids showed excellent agreement with those obtained by MG [20], indicating the capability of DQM in dealing with highly nonlinear partial differential equations.

\section{References}

1. Sander, G.C., Parlange, J.Y., Kuhnel, V., Hogarth, W.L., Lockington, D. and O'Kane, J.P.J. "Exact nonlinear solution for constant flux infiltration", $J$. Hydrol., 83, pp. 341-346 (1988).

2. Broadbridge, P. and White, I. "Constant rate rain fall infiltration: a versatile nonlinear model 1", Anal. Sol. Water. Res. Res., 24, pp. 145-154 (1988).

3. Tracy, F.T. "Three dimensional analytical solutions of Richard's equation for a box shaped soil sample with piecewise-constant head boundary conditions on the top", J. Hydrol., 336(3), pp. 391-400 (2007).

4. Menziani, M., Puganghi, S. and Vincenzi, M. "Analytical solutions of the linearized Richard's equation for discrete arbitrary initial and boundary conditions", $J$. Hydrol., 332(1-2) pp. 214-225 (2007).

5. Huang, R.Q. and Wu, L.Z. "Analytical solutions to 1-D horizontal and vertical water infiltration in saturated/unsaturated soils considering time-varying rainfall", J. Comp. Geotec., 39, pp. 66-72 (2012).

6. Haverkamp, R., Vauclin, M., Touma, J., Wiereng, P.J. and Vachaud, G. "A comparison of numerical simulation models for one-dimensional infiltration", $J$. Soil Soc. Am., 41, pp. 285-293 (1977).

7. G0ttardi, G. and Venutelli, M. "Two-dimensional finite-element groundwater flow model for saturatedunsaturated soils", J. Comput. Geosci., 27, pp. 179189 (2001).

8. Narado, N., Braud, I., Ross, P.J. and Haverkamp, R. "Assessment of an efficient numerical solution of the 1D Richard's equation on bare soil", J. Hydrol., 323(14), pp. 244-257 (2006).

9. Taheri Shahraiyani, H. and Ataei Ashtiani, B. "Comparison of finite difference schemes for water flow in unsaturated soils", Int. J. Aerosp. Mech. Eng., 3(1), pp. 1-5 (2009).

10. An, H., Ichikawa, Y., Tachikawa, Y. and Shiiba, M. "A new Iterative Alternating Direction Implicit (IADI) algorithm for multi-dimensional saturated-unsaturated flow", J. Hydrol., 408, pp. 127-139 (2011).

11. Bergamaschi, L. and Putti, M. "Mixed finite elements and Newton-type linearizations for the solution of 
Richards", Int. J. Num. Methods. Eng., 45, pp. 102546 (1999).

12. Gottardi, G. and Venutelli, M. "Two-dimensional finite-element groundwater flow model for saturatedunsaturated soils", J. Comput. Geosci., 27, pp. 179189 (2001).

13. He, X. and Ren, L. "An adaptive multiscale finite element method for unsaturated flow problems in heterogeneous porous media", J. Hydrol., pp. 56-70 (2009).

14. Ginting, V. "Time integration techniques for Richard's equation", Procedia Comput. Sci., 9. pp. 670-678 (2012).

15. Eymard, R., Gutnic, M. and Hilhorst, D. "The finite volume method for Richards' equation", J. Comput. Geosci., 12, pp. 259-294 (1999).

16. Manzini, G. and Ferraris, S. "Mass-conservative finite volume methods on 2-D unstructured grids for the Richards' equation", J. Adv. Water. Res., 27, pp. 1199-1215 (2004)

17. Misiats, O. and Lipnikov, K. "Second-order accurate monotone finite volume scheme for Richard's equation", J. Comput. Phys., 239, pp. 123-137 (2013).

18. Zambra, C.E., Dumbser, M., Toro, E.F. and Moraga, N.O. "A novel numerical method of high order accuracy for flow in unsaturated porous media", Int. $J$. Numer. Meth. Eng., 89, pp. 227-240 (2012).

19. Caviedes Voullieme, D., Garcia Navarro, P. and Murillo, J. "Verification, conservation, stability and efficiency of a finite volume method for the 1D Richards equation", J. Hydrol., 480, pp. 69-84 (2013).

20. Juncu, G., Nikola, A. and Popa, C. "Nonlinear multigrid methods for numerical solution of the unsaturated flow equation in two space dimensions", J. Transp. Porous. Med., 83, pp. 637-652 (2010).

21. Carr, E.J., Moroney, T.J. and Turner, I.W. "Efficient simulation of unsaturated flow using exponential time integration", J. Appl. Matt. Comput., 217(14), pp. 6587-6596 (2011).

22. Shu, C. Differential Quadrature and Its Application in Engineering, Springer, Singapore (2000).

23. Hashemi, M.R. and Hatam, F. "Unsteady seepage analysis using local radial basis function based differential quadrature method", Appl. Math. Model., 35, pp. 4934-4950 (2011).
24. Hu, Y.J., Zhu, Y.Y. and Cheng, C.J. "DQM for dynamic response of fluid-saturated visco-elastic porous media", Int. J. Solids Struct., 46, pp. 1667-1675 (2009).

25. Chen, R.P., Zhou, W.H., Wang, H.Z. and Chen, Y.M. "One-dimensional nonlinear consolidation of multilayered soil by differential quadrature method", $J$. Comput. Geotec., 32, pp. 358-369 (2009).

26. White, I. and Broadbridge, P. "Constant rate rainfall infiltration: a versatile nonlinear model 2", J. Appl. Sol. Water Res. Res., pp. 155-162 (1988).

27. Van Genuchten, M.T. "A closed-form equation for predicting the hydraulic conductivity of unsaturated soils", J. Soil Sci. Soc. Am., 44, pp. 892-898 (1980).

28. Belman, R., Kahef, B. and Casti, J. "Differential quadrature a technique for the rapid solution of nonlinear partial differential equations", J. Comput. Phys., 10, pp. 40-52 (1972).

29. Kuraz, M., Mayer, P., Leps, M. and Trpkosoa, D. "An adaptive time discretization of the classical and the dual porosity model of Richards' equation", $J$. Comput. App. Math., 233, pp. 3167-3177 (2010).

30. Asghari, A., Bagheripour, M.H. and Mollazadeh, M. "A generalized analytical solution for a nonlinear infiltration equation using the exp-function method", J. Scientia Iranica, 18(1), pp. 28-35 (2011).

31. Brooks, R.H. and Corey, A.T. Hydraulic Properties of Porous Media, Colorado State University, Fort Collins, Hydrology Paper, 3 (1964).

\section{Biographies}

Abdolhossein Baghlani is currently an Associate Professor of the Department of Civil and Environmental Engineering in Shiraz University of Technology, Shiraz, Iran. His areas of research and interest are optimization in civil engineering problems, hydraulic structures, sediment transport, computational fluid dynamics (CFD), and numerical simulations.

Jamshid Nikzad obtained his MSc degree in Hydraulic Structures. He is currently a PhD Student of Water Engineering in Najafabad Azad University. His areas of interest and research are water flow through porous media, numerical methods, sediment transport, and computational fluid dynamics. 